\title{
Effects of amphiphilic polypeptoid side chains on polymer surface chemistry and hydrophilicity
}

Mikayla E. Barry, ${ }^{1}$ Pinar Aydogan Gokturk, ${ }^{2}$ Audra J. DeStefano, ${ }^{3}$ Amanda K. Leonardi, ${ }^{4}$ Christopher K. Ober, ${ }^{4,5}$ Ethan J. Crumlin, ${ }^{2,6^{*}}$ and Rachel A. Segalman ${ }^{1,3 *}$

${ }^{1}$ Materials Department, University of California, Santa Barbara, California 93106, USA

${ }^{2}$ Advanced Light Source, Lawrence Berkeley National Laboratory, Berkeley, California 94720, USA

${ }^{3}$ Department of Chemical Engineering, University of California, Santa Barbara, California 93106, USA

${ }^{4}$ Department of Chemistry and Chemical Biology, Cornell University, Ithaca, New York 14853, USA

${ }^{5}$ Department of Materials Science and Engineering, Cornell University, Ithaca, New York 14853, USA

${ }^{6}$ Chemical Sciences Division, Lawrence Berkeley National Laboratory, Berkeley, California 94720, USA

*To whom correspondence should be addressed: ejcrumlin@lbl.gov, segalman@ucsb.edu

\section{Contents}

Polymer spectral components

Core level quantification

Soaked and unsoaked PDMS-peptoid samples comparison

Bilayer attenuation model of hydrated PDMS-peptoid surface

Quantification of water content

Synthesis

Polypeptoid synthesis

MALDI of polypeptoids

Thiol-ene "click" efficacy

\section{$\underline{\text { Polymer spectral components }}$}

Because of the complexity of the PEO-peptoid C 1s spectrum, the polypeptoid $\mathrm{N} 1 \mathrm{~s}$ amide $(\mathrm{N}-\mathrm{C}=$ $400.2 \mathrm{eV}$ ) was used to calibrate core level positions. ${ }^{1}$ For the PDMS-peptoid, the typical calibration of the siloxane oxygen $(\mathrm{O}-\mathrm{Si}=532.0 \mathrm{eV})^{2}$ was used due to the minimal polypeptoid content under dry conditions.

O 1s carbonyl and ether components at $531.7 \mathrm{eV}$ and $533.0 \mathrm{eV}$, respectively, are representative of literature values for peptides and PEO. ${ }^{1,2}$ Primary and secondary fluorinated hydrocarbons are visible at $294.0 \mathrm{eV}$ and $291.5 \mathrm{eV}$, with their F 1s counterparts at $689.4 \mathrm{eV}$. Carbonyl and ether-character carbon are at 288.1 and $286.7 \mathrm{eV}$, respectively, while nitrogen-bond carbon is at $286.1 \mathrm{eV}$. Minor amounts of aliphatic carbon from the side chain linker and polymer backbone can be seen at 285.1$285.2 \mathrm{eV}$ (PEO-peptoid) and 285.0 eV (PDMS-peptoid). For the PDMS-peptoid, silicon-bond oxygen is present at $532.0 \mathrm{eV}$ and silicon-bond carbon is present at $284.4 \mathrm{eV}$. 
(a)

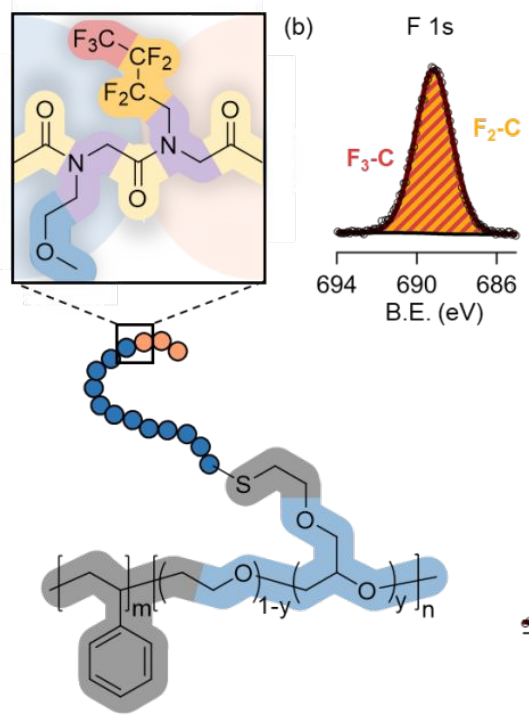



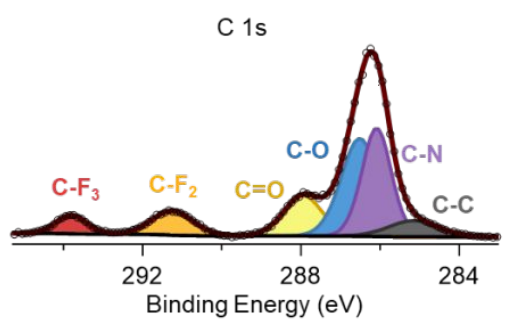

(c)
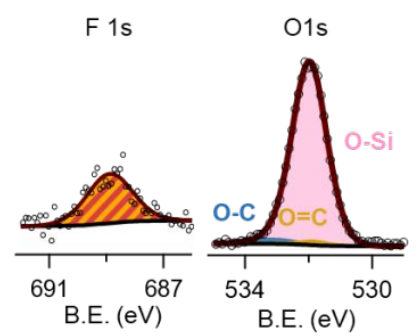

$\mathrm{N}$ 1s

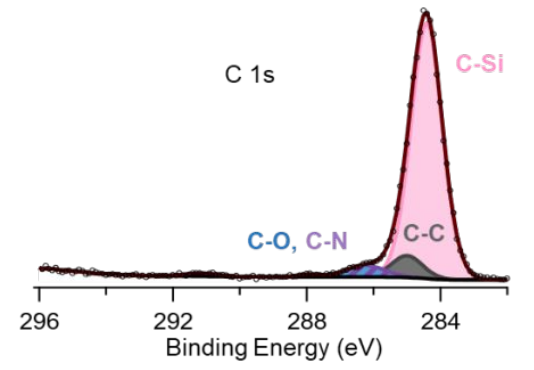

Figure S1. APXPS surface presentation of polypeptoid-modified PEO- and PDMS-based copolymers. (a) Representative chemical environments for the PEO-peptoid surface. (b) Relevant core levels for the PEOpeptoid surface, which is heavily composed of polypeptoid side chains. Measured under UHV with $150 \mathrm{eV}$ photoelectrons. (c) The PDMS-peptoid surface is mostly comprised of the siloxane polymer backbone. XP spectra were collected under 2 Torr Ar with $4 \mathrm{keV}$ photoelectrons.

\section{Core level quantification}

The amphiphilic polypeptoids contain a wide number of heteroatoms, such as the nitrogen- and fluorine- containing functional groups, that provide a number of non-carbon core levels to evaluate polypeptoid content at the surface. However, as seen in Figure 2, this diversity increases the number of species present in the carbon core levels. Cross-comparison between core levels is necessary for verification of functional group quantities (e.g., between $\mathrm{O} 1 \mathrm{~s}$ and $\mathrm{C}$ 1s for ether or carbonyl components).


(c) $\underset{U}{\mathrm{C} 1 \mathrm{~s}} 690 \mathrm{eV}$
$\mathrm{UHV}$

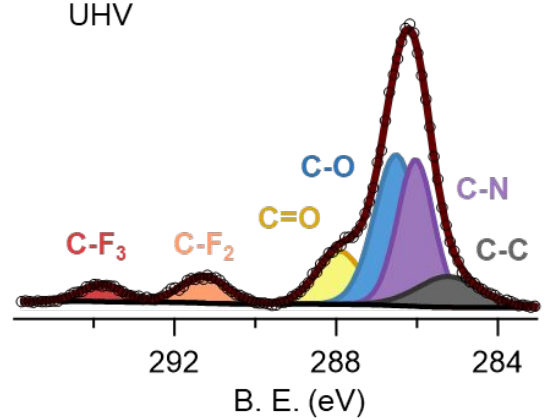

Figure S2. Ultrahigh vacuum spectra of the PS-PEO-peptoid copolymer surface at a single position. Large amounts of carbonyl-character oxygen in the $\mathrm{O} 1 \mathrm{~s}$ core level (a) and amide nitrogen in the $\mathrm{N}$ 1s core level (b) indicate polypeptoids are present in high quantities at the surface. These correlate quantitatively with their carbon neighbors in the $\mathrm{C}$ 1s spectrum (c), matched by color and label. 
Figure S2 depicts XP spectra of the PEO-peptoid surface at a single position. Quantification of the oxygen, nitrogen, and carbon core levels is consistent (Table S2), lending credibility to the fitting in the convolved, low-binding energy region of the $\mathrm{C}$ 1s spectrum: the total electron counts were found to be similar between oxygen and nitrogen species and their neighboring carbon equivalents in the $\mathrm{C} 1 \mathrm{~s}$ spectrum (matched by color in Figure S2). Component intensity of X-ray photoelectron spectra is quantitatively related to the atomic density $n$, photoelectron cross section $\sigma$, electron inelastic mean free path $l$, and sample thickness $d$, as indicated in Equation 1. ${ }^{3,4}$ For emitted electrons that are not elastically scattered, i.e., travel in a straight line, we can approximate the detected intensity by integrating over the exponential escape probability:

$$
I_{1} \approx n_{1} \sigma_{1} \int_{0}^{d} \exp \left(-\frac{z}{l_{1}}\right) d z=\varphi_{1} n_{1} \sigma_{1} l_{1}\left[1-\exp \left(-\frac{d}{l_{1}}\right)\right]
$$

For a sample with a single layer significantly thicker than the electron inelastic mean free path, the ratio between intensities (measured as region or component areas) can be simplified to

$$
\frac{I_{1}}{I_{2}}=\frac{n_{1} \sigma_{1} l_{1}}{n_{2} \sigma_{2} l_{2}} \cdot \frac{1-\exp \left(-\frac{d}{l_{1}}\right)}{1-\exp \left(-\frac{d^{\prime}}{l_{2}}\right)}=\frac{n_{1} \sigma_{1} l_{1}}{n_{2} \sigma_{2} l_{2}}
$$

The unknown intensity of a C 1s component can thus be solved for given the known heteroatom intensity and spectroscopic parameters. Table 1 depicts the values used to approximate the polymer component intensities in $\mathrm{C}$ 1s spectra. The relative atomic density of heteroatom and carbon pairs was determined according to the copolymer molecular formula. The resulting predictions were used to verify accuracy of $\mathrm{C} 1 \mathrm{~s}$ fits within $\sim 10 \%$ error.

\begin{tabular}{|c|c|c|c|c|c|}
\hline & Relative density & $\sigma(690 \mathrm{eV})$ & $l(690 \mathrm{eV})$ & $\sigma(4 \mathrm{keV})$ & $l(4 \mathrm{keV})$ \\
\hline O 1s & & $0.320 \mathrm{Mb}$ & $8.8 \AA$ & $2.11 \cdot 10^{-3} \mathrm{Mb}$ & $92.1 \AA$ \\
\hline $\mathrm{O}-\mathrm{C}$ & $n_{O-C}=(1 / 2) n_{C-O}$ & & & & \\
\hline $\mathrm{O}=\mathrm{C}$ & $n_{O=C}=n_{C=O}$ & & & & \\
\hline N 1s & & $0.208 \mathrm{Mb}$ & $12.8 \AA$ & $1.21 \cdot 10^{-3} \mathrm{Mb}$ & $95.0 \AA$ \\
\hline $\mathrm{N}-\mathrm{C}$ & $n_{N-C}=n_{C-N}$ & & & & \\
\hline F 1s & & -- & -- & $3.26 \cdot 10^{-3} \mathrm{Mb}$ & $88.6 \AA$ \\
\hline $\mathrm{F}-\mathrm{C}$ & $n_{F-C}=(7 / 3) n_{C-F}$ & & & & \\
\hline C 1s & & $0.121 \mathrm{Mb}$ & $16.3 \AA$ & $6.57 \cdot 10^{-4} \mathrm{Mb}$ & $97.5 \AA$ \\
\hline
\end{tabular}

Table 1. Parameter values for verification of $\mathrm{C} 1 \mathrm{~s}$ spectral components. 
Table 2. Representative prediction and verification of $\mathrm{C} 1 \mathrm{~s}$ fits based on heteroatom core levels. Listed spectra are from high vacuum conditions at $690 \mathrm{eV}$ at the same position.

\begin{tabular}{|cccccc|}
\hline & $\begin{array}{c}\text { Heteroatom } \\
\text { fit }\end{array}$ & RSF & $\begin{array}{c}\text { Predicted } \\
\text { C 1s }\end{array}$ & C 1s fit & $\begin{array}{c}\text { Percent } \\
\text { difference }\end{array}$ \\
\hline $\mathrm{N}-\mathrm{C}$ & 825,848 & 1.49 & $1,230,771$ & $1,288,201$ & $4.6 \%$ \\
$\mathrm{O}=\mathrm{C}$ & 923,930 & 0.704 & 650,655 & 582,487 & $11.1 \%$ \\
$\mathrm{O}-\mathrm{C}$ & $1,071,817$ & 1.41 & $1,507,478$ & $1,576,313$ & $4.5 \%$ \\
\hline
\end{tabular}

Soaked and unsoaked PDMS-peptoid samples
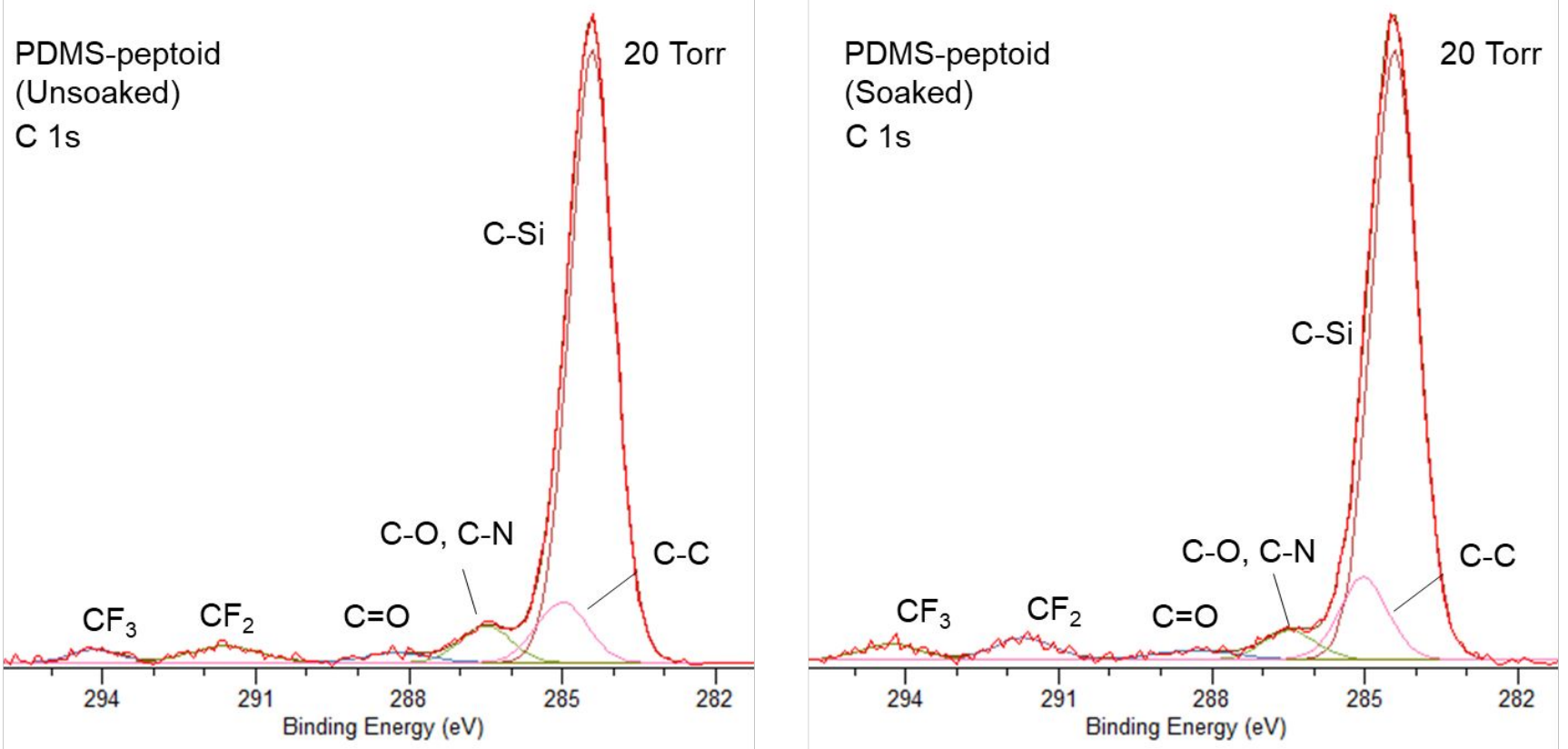

Figure S3. Carbon 1s spectra show similar composition between soaked and unsoaked samples.

$\underline{\text { Polypeptoid-modified polymer component spectra }}$
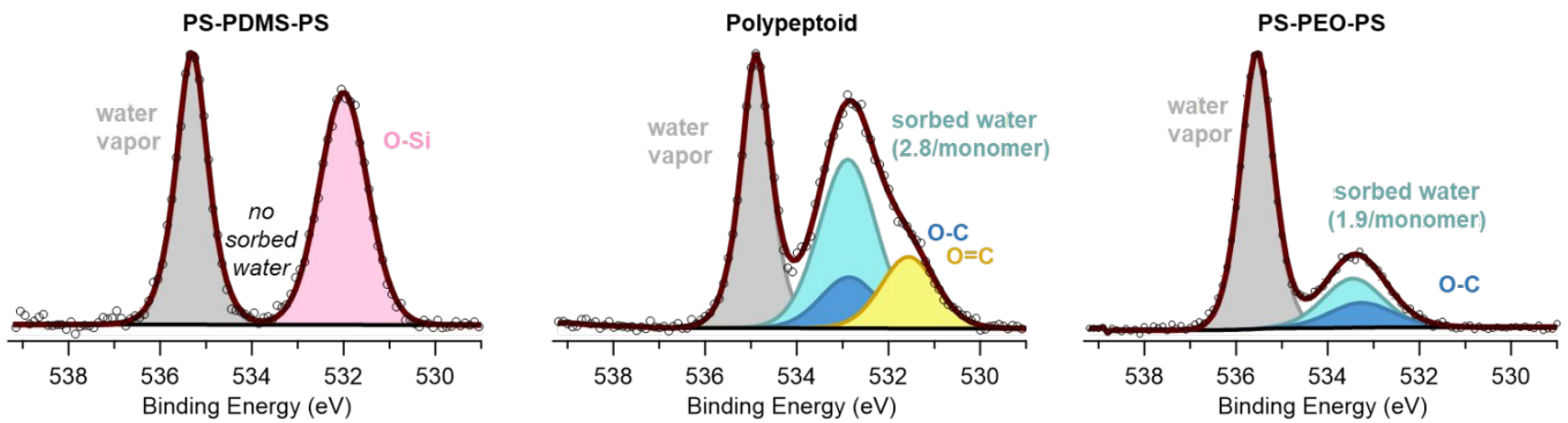

Figure S4. Water sorption onto PDMS, polypeptoid, and PEO surfaces show different water affinities in terms of sorption. 
Bilayer attenuation model of hydrated PDMS-peptoid surface

For the PDMS-peptoid system, relatively small amounts of water were detected compared with the overall polymer. A two-layer XPS attenuation model served as a metric for observing the maximum amount of water at the surface. From literature, the thickness $d_{\mathrm{w}}$ of a water layer adsorbed onto (but not sorbed into) a surface can be approximated using the components' intensities $\left(I_{p}, I_{w}\right)$, their photoionization cross sections $(\sigma)$, atomic density $(N)$, and inelastic mean free paths $(l){ }^{3}$

$$
d_{w}=l_{w} \ln \left(\left[1-\exp \left(-\frac{\mathrm{d}_{\mathrm{p}}}{l_{\mathrm{p}}}\right)\right]\left[\frac{I_{w} N_{p} \sigma_{p} l_{p}}{I_{p} N_{\mathrm{w}} \sigma_{\mathrm{w}} l_{\mathrm{w}}}\right]+1\right)
$$

Applying this model to the 20 Torr O 1s spectrum, the PDMS-peptoid surface held a $4.5 \AA$ layer of water ( $\sim 1.5$ monolayers of water). Table 3 shows the parameter values used for the calculation.

Table 3. Parameter values for bilayer attenuation model

\begin{tabular}{|lcccc|}
\hline & $\mathbf{I}$ & $\mathbf{N ~}\left(\mathbf{c m}^{-3}\right)^{*}$ & $\boldsymbol{\sigma}(\mathbf{M b})$ & $\boldsymbol{l}(\AA))^{5,6}$ \\
\hline Water & $20,629.4$ & $3.34 \mathrm{E}+22$ & $2.11 \cdot 10^{-3} \mathrm{Mb}$ & $103.8 \AA$ \\
Polymer (PDMS) & $117,922.5$ & $7.83 \mathrm{E}+21$ & $2.11 \cdot 10^{-3} \mathrm{Mb}$ & $112.1 \AA$ \\
\hline
\end{tabular}

*Atomic density was determined based on bulk material density.

\section{Quantification of water content}

Because the PDMS-peptoid water content is contained only within the polypeptoid, the carbonyl and ether oxygen components were used within the $\mathrm{O} 1 \mathrm{~s}$ to identify the ratio between sorbed water and polypeptoid oxygen (1.54). The oxygen content of a polypeptoid monomer averages to $1.86 \mathrm{O}$ per polypeptoid monomer ( 1 per fluorinated, 2 per ether), and therefore sorbed water exists at a ratio of 2.83 water per polypeptoid monomer. The amounts of sorbed water in polypeptoid and PS-PEO-PS surfaces were found using the same methods.

Quantification of water content in the PEO-peptoid surface requires untangling of the z-dependent surface composition. Depth profiling indicates larger amounts of polypeptoid within the top $5 \mathrm{~nm}$ of the surface. By linearly fitting the excess fluorinated carbon content, estimates of the polypeptoid and PEO monomer fractions were made for the skin and bulk regions. A linear fit was chosen as the simplest gradient estimate since all measurements are also affected by the exponentially decaying depth-dependent signal intensity (in addition to the depth-dependent composition).
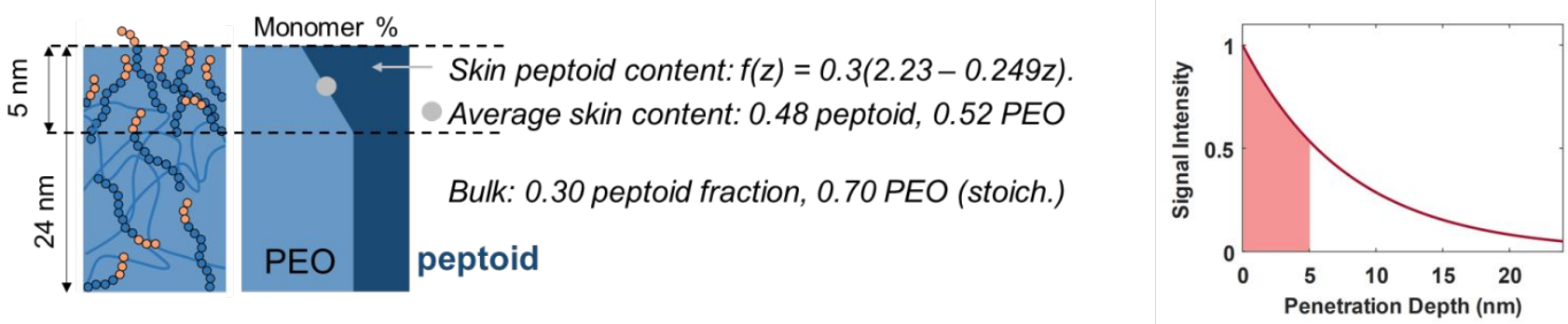
The amount of bound water per monomer differs for the PEO $\left(w_{P E O}=1.9\right)$ and polypeptoid $\left(w_{p e p}=\right.$ $2.80)$ components. Therefore, the estimated net amount of water per monomer depends on the relative fractions in the skin and bulk layers:

$$
\begin{aligned}
& W_{\text {skin }}=0.48 w_{\text {pep }}+0.52 w_{P E O}=2.33 \text { water molecules/polymer monomer } \\
& W_{\text {bulk }}=0.30 w_{\text {pep }}+0.70 w_{P E O}=\mathbf{2 . 1 7} \text { water molecules/polymer monomer }
\end{aligned}
$$

The model can be verified by comparing the amount of sorbed water relative to the polymer in the $\mathrm{O}$ 1s spectrum with the model prediction. The amount of oxygen molecules per monomer differs between the skin and bulk layers due to changes in polypeptoid content. Given an average of $1.86 \mathrm{O}$ per polypeptoid monomer ( 1 per fluorinated, 2 per ether) and 1.03 per polymer backbone ( 1 per EO, 2 per AGE), the skin and bulk consist of 1.43 and 1.28 oxygen atoms per polymer monomer, respectively. Dividing $W_{\text {skin }}$ and $W_{\text {bulk }}$ by each layer's oxygen content per monomer yields the ratio of water molecules per polymer oxygen.

It is important to note that the skin layer contributes approximately $48.9 \%$ of total signal from the polymer surface. Thus, weighting both layers' water composition yields an estimate of 1.66 water molecules per polymer oxygen for the $\mathrm{O} 1 \mathrm{~s}$ spectrum, a value that is close to the 1.7 ratio found experimentally.

\section{Polypeptoid synthesis}

Solid-phase synthesis was conducted with minimal modifications to published procedures on a Prelude synthesizer (Protein Technologies, Inc.) ${ }^{7-9}$ Rink amide resin ( $0.40 \mathrm{mmol} \mathrm{g}{ }^{-1}$ loading) was first deprotected with 20\% 4-methylpiperidine solution in dimethylformamide (DMF) for 20 minutes, before washing in DMF. The free amine chain end was acylated with $0.6 \mathrm{M}$ bromoacetic acid and 0.4 M N,N-diisopropylcarbodiimide (DIC) in DMF for $20 \mathrm{~min}$. Following washing in DMF, nucleophilic displacement of the bromine was performed with either 2-methoxyethylamine or $1 \mathrm{H}, 1 \mathrm{H}$ heptafluorobutylamine to yield ether or fluorinated peptoid monomers, respectively. A concentration of $1 \mathrm{M}$ in DMF was used for both 2-methoxyelthylamine and 2,2,3,3,4,4,4-heptafluorobutylamine. The heptafluorobutylamine additions proceeded for 3 hours at $50{ }^{\circ} \mathrm{C}$ and the methoxyethylamine additions proceeded for 1 hour at room temperature. After the monomer solution was drained from the resin using the Prelude, the resin was again washed with DMF before repeating additions. The polypeptoid was functionalized with a thiol endgroup using S-trityl-3-mercaptopropionic acid and N,N'diisopropylcarbodiimide (DIC) (both $0.4 \mathrm{M}$ in DMF). $2 \mathrm{~mL}$ of solution per $50 \mu \mathrm{mol}$ peptoid were added, bubbled with $\mathrm{N}_{2}$ for 30 minutes, and washed with DMF.

Polypeptoids were cleaved from the solid support using a cocktail of trifluoroacetic acid : dicholoromethane : triisopropylsilane: water $(47.5: 47.5: 2.5: 2.5, \mathrm{v} / \mathrm{v} / \mathrm{v} / \mathrm{v})$ for 20 minutes, a process which simultaneously deprotected the thiol. The resin was filtered and rinsed with dichloromethane and the collected solution was dried under vacuum and lyophilized from water and acetonitrile $(1: 1$, $\mathrm{v} / \mathrm{v})$. MALDI was performed to confirm the completion of the synthesis. 


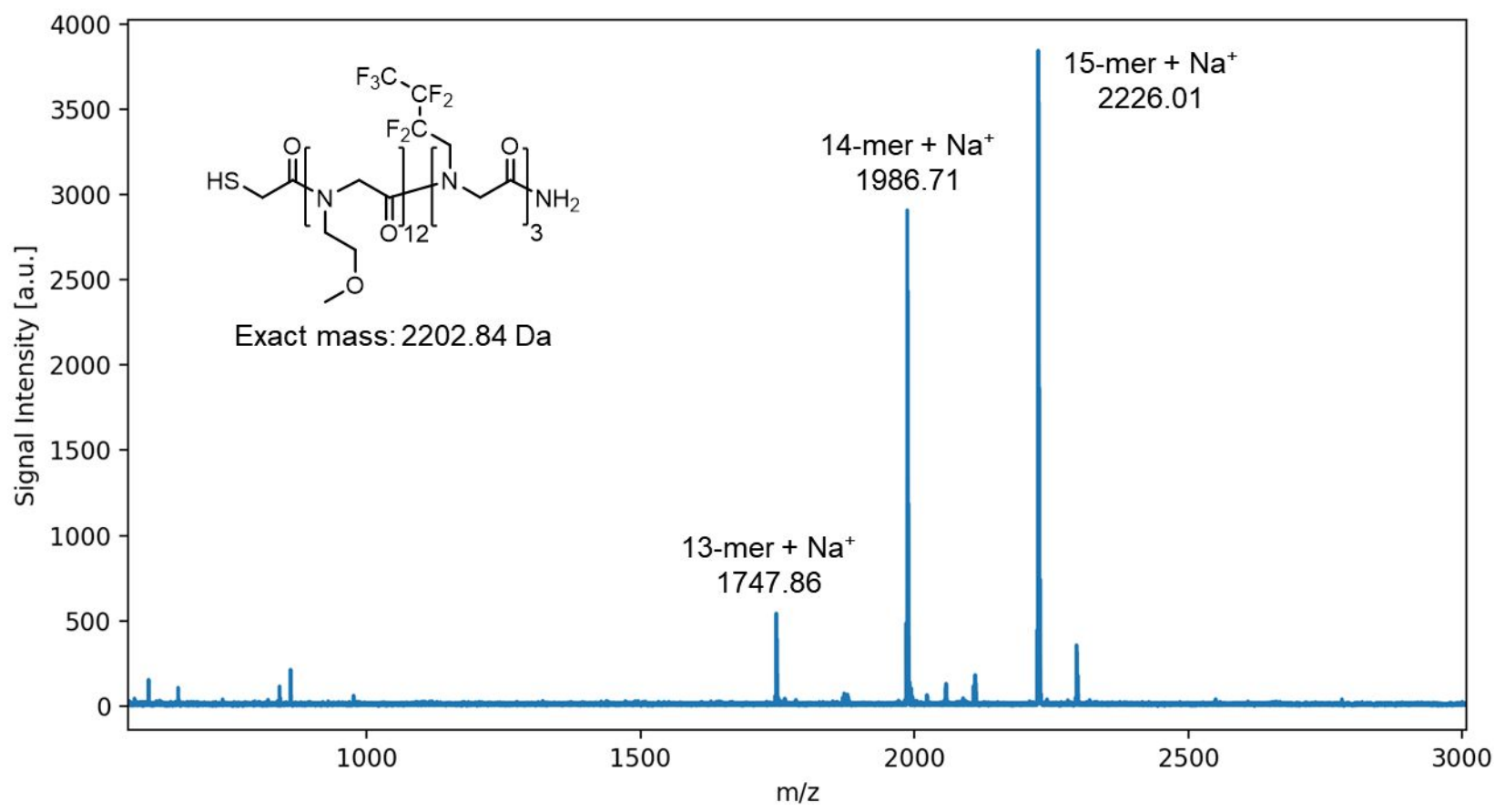

Figure S4. MALDI trace of the synthesized polypeptoid. The desired 15-mer polypeptoid was shown to be a majority product, with a detected mass of $2226.01 \mathrm{Da}\left(+\mathrm{Na}^{+}\right)$. A moderate amount of 14-mers was also detected containing two of three fluorinated monomers, and a small amount of 13-mers with only one fluorinated monomer. 


\section{$\underline{\text { Click efficacy }}$}

a) PEO-peptoid click attachment



b) PEO-peptoid click attachment
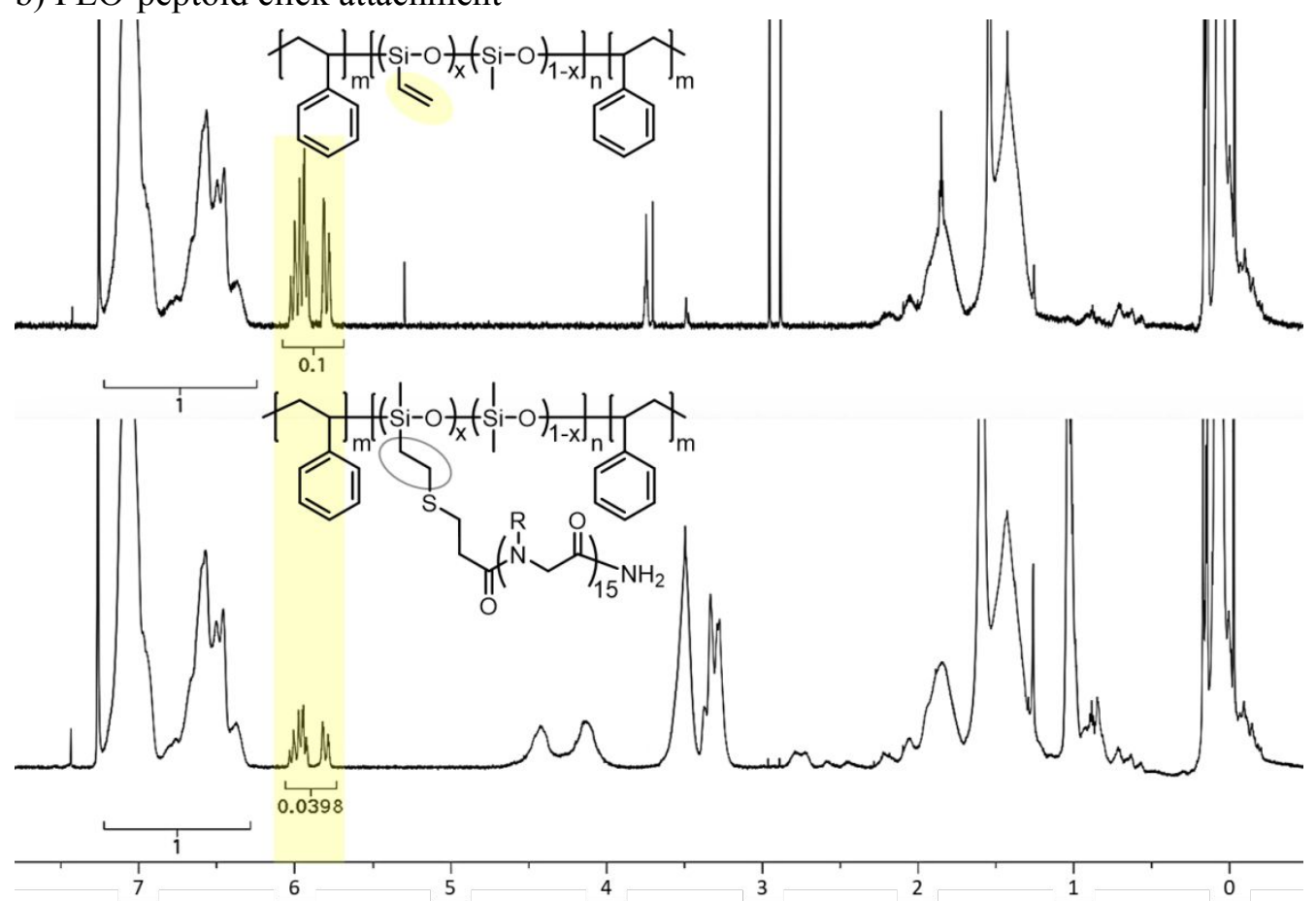

Figure S5. Reduction in allyl and vinyl components were indicative of polypeptoid attachment during thiol-ene click reaction. Due to steric hindrance resulting from the trivinyl siloxane comonomer, the PDMS-peptoid copolymer reached approximately 60\% completion. This represents attachment at approximately two of the three vinyl groups per comonomer. 
References

(1) Gervais, M.; Douy, A.; Gallot, B.; Erre, R. Surface Analysis of Lipopeptides Using X-Ray Photoelectron Spectroscopy: I. Lipopeptides with Polysarcosine Peptidic Chains. J. Colloid Interface Sci. 1988, 125 (1), 146-154. https://doi.org/10.1016/0021-9797(88)90063-X.

(2) G. Beamson; D. Briggs. High Resolution XPS of Organic Polymers: The Scienta ESCA300 Database; Wiley-VCH, 1992.

(3) Hofmann, S. Quantitative Analysis (Data Evaluation). In Auger-and X-Ray Photoelectron Spectroscopy in Materials Science: A User-Oriented Guide; Springer Science \& Business Media, 2012; pp 77-201.

(4) Trotochaud, L.; Head, A. R.; Pletincx, S.; Karslığlu, O.; Yu, Y.; Waldner, A.; Kyhl, L.; Hauffman, T.; Terryn, H.; Eichhorn, B.; Bluhm, H. Water Adsorption and Dissociation on Polycrystalline Copper Oxides: Effects of Environmental Contamination and Experimental Protocol. J. Phys. Chem. B 2018, 122 (2), 1000-1008. https://doi.org/10.1021/acs.jpcb.7b10732.

(5) Shinotsuka, H.; Da, B.; Tanuma, S.; Yoshikawa, H.; Powell, C. J.; Penn, D. R. Calculations of Electron Inelastic Mean Free Paths. XI. Data for Liquid Water for Energies from 50 EV to 30 KeV. Surf. Interface Anal. 2017, 49 (4), 238-252. https://doi.org/10.1002/sia.6123.

(6) Cumpson, P. J. Estimation of Inelastic Mean Free Paths for Polymers and Other Organic Materials: Use of Quantitative Structure-Property Relationships. Surf. Interface Anal. 2001, 31 (1), 23-34. https://doi.org/10.1002/sia.948.

(7) van Zoelen, W.; Buss, H. G.; Ellebracht, N. C.; Lynd, N. A.; Fischer, D. A.; Finlay, J.; Hill, S.; Callow, M. E.; Callow, J. A.; Kramer, E. J.; Zuckermann, R. N.; Segalman, R. A. Sequence of Hydrophobic and Hydrophilic Residues in Amphiphilic Polymer Coatings Affects Surface Structure and Marine Antifouling/Fouling Release Properties. ACS Macro Lett. 2014, 3 (4), 364 368. https://doi.org/10.1021/mz500090n.

(8) Patterson, A. L.; Wenning, B.; Rizis, G.; Calabrese, D. R.; Finlay, J. A.; Franco, S. C.; Zuckermann, R. N.; Clare, A. S.; Kramer, E. J.; Ober, C. K.; Segalman, R. A. Role of Backbone Chemistry and Monomer Sequence in Amphiphilic Oligopeptide- and OligopeptoidFunctionalized PDMS- and PEO-Based Block Copolymers for Marine Antifouling and Fouling Release Coatings. Macromolecules 2017, 50 (7), 2656-2667. https://doi.org/10.1021/acs.macromol.6b02505.

(9) Barry, M. E.; Davidson, E. C.; Zhang, C.; Patterson, A. L.; Yu, B.; Leonardi, A. K.; Duzen, N.; Malaviya, K.; Clarke, J. L.; Finlay, J. A.; Clare, A. S.; Chen, Z.; Ober, C. K.; Segalman, R. A. The Role of Hydrogen Bonding in Peptoid-Based Marine Antifouling Coatings. Macromolecules 2019, 52 (3), 1287-1295. https://doi.org/10.1021/acs.macromol.8b02390. 